\title{
Manejo anestésico en una mujer con malformación de Arnold-Chiari tipo II residual
}

\author{
JUAN MANUEL RODRÍGUEZ Z. ${ }^{1}$, JUAN FRANCISCO MONZÓN F. ${ }^{2}$, OFELIA JAM M. ${ }^{1}$, \\ SERGIO MORENO J. ${ }^{3}$, JUAN LAGARDA C. ${ }^{4}$
}

\author{
Centro Médico ABC Servicio de Anestesiología. \\ Instituto Nacional de Neurología Médico en Servicio Social. \\ Centro Médico ABC Servicio de Neurocirugía. \\ Centro Médico ABC Servicio de Anestesiología, Jefe de Área Médica Nivel Central IMSS.
}

\begin{abstract}
Anesthetic management in a woman with residual Arnold-Chiari malformation Type II
\end{abstract}

Background: The Arnold-Chiari malformation (ACM) is a group of congenital abnormalities of the hindbrain and the spinal cord and is characterized by herniation of the cerebellum, kinking of the medulla oblongata and hydrocephalus, the anesthetic management Is complicated due to the anatomic and physiologic alterations. Case description: A 23 years old woman with Arnold-Chiari type II malformation, and perforated appendicitis. Conclusions: The present case demonstrates that patients with partially corrected ACM type II, restrictive lung disease due to scoliosis and perforated appendicitis delivery require an interdisciplinary team approach, diligent preparation, and skilled physicians.

Key words: Anesthetic management, Arnold-Chiari malformation, pneumonia, sepsis, appendicitis.

\section{RESUMEN}

Antecedentes: La malformación de Arnold-Chiari (ACM) es un grupo de anomalías congénitas del cerebro posterior y la médula espinal que se caracteriza por la herniación del cerebelo, retorcimiento del bulbo raquídeo e hidrocefalia, el manejo anestésico se complica debido a la anatomía y alteraciones fisiológicas. Descripción del caso: Una mujer de 23 años con Arnold-Chiari tipo II malformación y apendicitis perforada. Conclusiones: El presente caso demuestra que los pacientes con corregido parcialmente ACM tipo II, enfermedad pulmonar restrictiva debido a la escoliosis y la entrega apendicitis perforada requieren un enfoque multidisciplinario en equipo, preparación diligente, y los médicos cualificados.

Palabras clave: El manejo anestésico, Arnold-Chiari, neumonía, sepsis, apendicitis.

\author{
Autor Responsable: \\ Dr. Juan Manuel Rodríguez Zepeda \\ Anestesiólogo Pediatra, Centro Médico ABC, México, D.F. \\ Teléfonos: 0445514517480 \\ E-mail: juanma51@hotmail.com \\ Correspondencia a: \\ juanma51@hotmail.com \\ Campeche 175 Interior 802 Colonia Roma CP 06700 \\ Delegación Cuauhtemoc Ciudad de México.
}




\section{Introducción}

La malformación de Arnold-Chiari ofrece una serie de retos anestésicos. Dada su incidencia baja en centros de alta especialidad, donde se realiza y fundamenta su diagnóstico, es difícil que el anestesiólogo se encuentre familiarizado con las repercusiones anestésicas que se presentan a continuación. Enumeramos una serie de consideraciones anestésicas y anatómicas que se deben tomar en cuenta para realizar un procedimiento anestésico en los pacientes con malformación de Arnold-Chiari.

La malformación de Arnold-Chiari es una malformación congénita muy poco frecuente, consiste en una alteración anatómica de la base del cráneo con un descenso de las amígdalas cerebelosas por debajo del foramen magno un mínimo de 5 milímetros en ausencia de espina bífida ${ }^{1,3,5,12}$.

Aunque no existe una prevalencia exacta de la enfermedad de Arnold-Chiari se estima que afecte entre el 6 y el $8 \%$ de la población mundial (Feder, septiembre de 2010).

Hay cuatro formas tradicionales de malformaciones de Chiari que suponen diferentes grados de afectación del romboencéfalo ${ }^{1}$.

La malformación de Arnold-Chiari tipo I es de origen congénito y secundaria a defectos en el desarrollo del mesodermo ${ }^{7}$, o adquirida secundaria a cortocircuitos lumboperitoneales o tumores de fosa posterior ${ }^{5}$.

El tipo II también llamada malformación clásica, involucra la extensión de los tejidos cerebeloso y del tallo cerebral dentro del foramen magno se debe a una malformación congénita del cierre del tubo neural, se caracteriza por herniación caudal a través del foramen magno del vermis cerebeloso, tronco del encéfalo y cuarto ventrículo. Se asocia con mielomeningocele en un $100 \%$ e hidrocefalia ${ }^{7,12}$.

La malformación tipo III es rara se debe también a un defecto congénito, el cual se caracteriza por un encefalocele occipital con parte de las anomalías del Chiari tipo II. En éste el cerebelo y el tallo cerebral sobresalen o se hernian a través del foramen magno hacia la médula espinal. Parte del cuarto ventrículo del cerebro, tiene alta tasa de mortalidad temprana y en los sobrevivientes causa graves daños neurológicos irreversibles $^{3,7,12,14}$.
La malformación tipo IV es una manifestación rara, consiste en aplasia o hipoplasia del cerebelo asociada con aplasia de la tienda del cerebelo, así como malformaciones en el tallo y cerebro $^{7,12}$.

La causa exacta de una malformación de Chiari aún se desconoce, se cree que un problema durante el desarrollo fetal puede ocasionar formación anormal del encéfalo ${ }^{3,12,7}$.

Existe gran variabilidad clínica entre las personas afectadas (asintomáticas, manifestaciones clínicas inespecíficas o signos neurológicos graves) $)^{3,12,7}$.

La heterogeneidad en las características de presentación, el diagnóstico en la consulta de atención primaria puede verse dificultado. En la actualidad la resonancia magnética ha revolucionado el diagnóstico precoz de estas malformaciones, siendo la piedra angular en el diagnóstico de estas malformaciones ${ }^{7,3,1}$.

El tratamiento aceptado de los pacientes diagnosticados de la malformación de ArnoldChiari es la descompresión del foramen magno con craneotomía suboccipital y resección del arco posterior del atlas, durotomía, liberación de adherencias aracnoideas y ascenso de las amígdalas y plastía dural ${ }^{1,10}$.

Dada las características anatómicas la malformación de Arnold-Chiari dificulta el manejo anestésico desde el manejo de la vía aérea, siendo una vía aérea difícil en la mayoría de los casos. La anestesia general con intubación endotraqueal es una técnica segura para los procedimientos quirúrgicos para estos pacientes, se debe planificar una intubación difícil por las posibles alteraciones esqueléticas asociadas, también se deben extremar las precauciones para evitar aumentos de la presión intracraneal; por ello, se debe utilizar fármacos que no la aumenten, así como maniobras de intubación con fibroscopio o con vídeo laringoscopios y para la extubación en un plano lo suficientemente profundo de anestesia, sin que se afecte la oxigenación ${ }^{21}$.

Una de las metas para la anestesia en los pacientes con Arnold-Chiari, es mantener estable la presión del LCR intracraneal y del canal medular, para evitar una progresión o agravamiento del cuadro, lo que es concordante con la patogenia de la enfermedad ${ }^{20}$.

Es necesario recordar que debido al compromiso neuromuscular, es peligroso utilizar 
succinilcolina para la intubación por el riesgo de hiperkalemia secundaria a la proliferación de receptores de acetilcolina inmaduros. El resto de los relajantes neuromusculares pueden presentar una mayor duración del efecto, unido a las posibles alteraciones de la mecánica ventilatoria, ya sea por compromiso de la musculatura respiratoria o por alteraciones de la caja torácica ${ }^{22}$.

Durante el postoperatorio es importante mantener la estrecha monitorización cardiorrespiratoria porque la inflamación secundaria a la cirugía puede producir efectos deletéreos en estos sistemas debido a sus antecedentes neurológicos así como de malformación Arnold-Chiari tipo II, a su neuropatía restrictiva por escoliosis así como antecedentes de infecciones pulmonares.

A continuación presentamos el siguiente caso.

\section{Reporte de caso}

Paciente femenino de 23 años originaria de Chile, reside en el DF desde el año 2011, paciente con antecedentes heredofamiliares de importancia presencia de hipertensión arterial sistémica en ambos padres. Habita en casa rentada la cual cuenta con todos los servicios básicos de urbanización, tabaquismo, alcoholismo y toxicomanías negadas. Sedentarismos con limitación de la marcha por malformación de columna secundaria a mielomeningocele, requiere oxígeno suplementario. Dentro de los antecedentes patológicos de intereses se encuentra: Malformación de Arnold-Chiari, mielomeningocele, neumonía de focos múltiples de repetición, último internamiento hace 1 año, no requirió ventilación mecánica, hidrocefalia con colocación de válvula ventrículo-peritoneal inicialmente a los 21 días de nacida con recolocación a los 5 años de edad, presencia de rotoescoliosis, cistostomía a los 6 años de edad y colocación de osteosíntesis en columna a los 3 años; se le realizan 3 cirugías 2 de ellas con presencia de estafilococo metilcilino resistente. Transfusiones en cirugía de colocación de válvula ventrículo peritoneal sin reacción a la misma. Niega alergias.

Ingresa al centro médico $\mathrm{ABC}$ el día 06/09/12 por presentar su padecimiento con pico febril de 40,6 grados $24 \mathrm{~h}$ antes, relacionado con tos seca. Por continuar con sintomatología acude al servi- cio de urgencias.

A la exploración física se encuentra paciente femenino con edad cronológica mayor a la aparente con escoliosis con adecuado estado de hidratación y coloración de piel y mucosas. Alerta, orientada, cooperadora con TA 91/56 FC 159 FR 24, Temp. 38,5 sp02 81\% (oxígeno-dependiente), Nistagmus horizontal no fatigable, con hiperemia faríngea y amígdalas hipertróficas GII, con cuello corto, con limitación de la movilidad, cifoescoliosis que modifica anatomía de tórax, campos pulmonares con estertores finos bilaterales, sin integrar síndrome pleuropulmonar. Extremidades hipotróficas con rotación externa de miembro pélvico derecho con sensibilidad y fuerza ausente en ambas extremidades.

Leucocitos 9,3, Hb 13,4, HTO 43,3, Pla 252, TP 13,4, TTPA 25, Gluc 115, BUN 19, Cre 0,5, $\mathrm{Na} 141, \mathrm{~K} 3,4, \mathrm{Cl} 102, \mathrm{C} 02$ 23,7.

EKG: ritmo sinusal, FC $130 \mathrm{X}^{\prime} \mathrm{AQRS} 65^{\circ}$, PR 11, infra desnivel ST V2, V3, V4, relacionada con anatomía con S1Q3T3 con datos de sobrecarga del ventrículo derecho.

De los estudios de gabinete realizados se realiza Ecocardiograma: ventrículo izquierdo de dimensiones y movilidad normales FEVI 65\%, función diastólica normal, ventrículo derecho de dimensiones y movilidad normales, válvulas normofuncionales, presión pulmonar normal, PSAP $22 \mathrm{mmHg}$, pericardio normal.

TAC simple toracoabdominal en la que se observa alteración importante de la anatomía en cuestión esquelética, presencia de atelectasia laminar izquierda y apendicolito con plastrón periapendicular. Se diagnostica apendicitis en fase aguda III/IV se decide intervención quirúrgica de emergencia.

Debido a lo anterior se realiza tratamiento quirúrgico laparoscópico convirtiéndola a laparotomía debido a anatomía de la paciente y a densidad de las adherencias así como a los hallazgos topográficos se decidió realizar laparotomía en la cual se encuentra apéndice en una localización retroperitoneal retrocecal y paravertebral perforada con contenidos de dos fecalitos en su interior, tiempo quirúrgico de $4,5 \mathrm{~h}$ con 10 $\mathrm{ml}$ de sangrado y $50 \mathrm{ml}$ de diuresis.

Durante el procedimiento la paciente tiene un riesgo quirúrgico ASA III, se realiza inducción con fentanilo $70 \mathrm{mcg}$, rocuronio $30 \mathrm{mcg}$, propofol $120 \mathrm{mg}$, xilocaina $10 \mathrm{mg}$. 
Vía área difícil entubada con cánula \#7 tubo endotraqueal Bullard 6,5, intubado al primer intento, protección ocular bilateral, mantenimiento con sevoflurano 2 vol \% dexmedetomidina en infusión $3 \mathrm{mcg} / \mathrm{kg} / \mathrm{h}$, ventilación con presión inspirada $19 \mathrm{~cm} \mathrm{H}_{2} 0$, FR $18 \mathrm{X}^{\prime}$ 1: E 1:3 PEEP $5 \mathrm{~cm} \mathrm{H}_{2} 0$, Ppivo $17 \mathrm{mmH}_{2} 0$. Mantenimiento hemodinámicamente estable TAM $>60 \mathrm{mmHg}$, $\mathrm{SaO}_{2} 85-99 \%$ FC $80-115 \mathrm{X}^{\prime}$, se traslada a terapia intensiva sin extubar con FC: 86 x' $\mathrm{SaO}_{2} 99$ TA 82/51 debido a sus antecedentes neurológicos así como de malformación Arnold-Chiari tipo II, a su neuropatía restrictiva por escoliosis así como antecedentes de infecciones pulmonares. La paciente permanece 4 días en el servicio de terapia intensiva. En el primer día de estancia en terapia presenta extubación fortuita por parte de ella a pesar de estar bajo sedación por lo que se asistió con mascarilla y se vigiló trabajo respiratorio, se revirtió efecto de fentanilo y respondió favorablemente manteniendo $\mathrm{FiO}_{2}$ de $40 \%$ saturaciones periféricas de $94 \%$, no datos de bronco espasmos, se encuentra la presencia de SRIS (taquicardia $100 \mathrm{X}^{\prime}$ leucocitosis 20,1) se maneja cobertura antimicrobiana con meropenem, sangrado por la herida quirúrgica mínimo mantiene 1,1 $\mathrm{ml} / \mathrm{kg} / \mathrm{h}$ de diuresis, balance positivo, se reanimó con normosol (bicarbonato debido a la presencia de acidosis metabólica hiperclorémica anion gap 10 , presentó $\mathrm{CO}_{2}$ total de 20,6 y Na 145 disminuye aporte de bicarbonato.

Dada su mejoría clínica finalmente se egresa del hospital el día 13/09/2012 con signos vitales normales, abdomen blando, depresible sin datos de irritación peritoneal, peristalsis presente, diuresis normal, canaliza gases, tolera dieta normal, no incremento en trabajo respiratorio.

\section{Discusión}

La enfermedad o el síndrome de Arnold-Chiari ofrecen una serie de retos anestésicos. Dada su incidencia baja en centros de alta especialidad donde se realiza y fundamenta su diagnóstico, es difícil que el anestesiólogo se encuentre familiarizado con las repercusiones anestésicas que presenta. A continuación enumeramos una serie de consideraciones anestésicas que hay que tomar en cuenta cuando el anestesiólogo se encuentre con estos retos:
Valoración Pre anestésica: Por ser una alteración de tipo congénito. Es importante hacer énfasis en conjuntar la mayor información posible de este tipo de pacientes desde el punto de vista perinatal como es edad gestacional, peso al nacimiento, apgar. De igual forma es importante indagar el tipo de enfermedad con la cual nos enfrentamos sea I, II, III o IV. Si el paciente ha recibido tratamiento multidisciplinario o no. Y tomar muy en cuenta la serie de comorbilidades de las cuales el síndrome puede venir acompañado. Si el tiempo lo permite solicitar valoración por neurocirugía, cardiología, genética entre otros. Considere enfáticamente ayuno y la posibilidad latente de aspiración a pesar de éste.

Técnica Anestésica: El uso de anestesia regional no está contraindicado, sin embargo, existen pocos reportes de su uso enfatizando que el bloqueo peridural puede ser utilizado de manera lenta a fin de no repercutir en el estado hemodinámico de estos pacientes. No se recomienda el bloqueo subaracnoideo. La anestesia general balanceada podría ser hasta este momento la técnica de elección siempre y cuando se mantenga una estabilidad hemodinámica sin cambios bruscos en la tensión arterial y por ende la presión del LCR.

Preparación de equipo anestésico y logística perioperatoria: Es de suma importancia el contar con equipo adecuado para afrontar las alteraciones anatómicas y repercusiones fisiopatológicas que estos pacientes pudiesen presentar, como por ejemplo contar con un carro de vía aérea difícil.

Premedicación: Quizá la ansiolisis sea un punto a considerar ya que la disminución en la ansiedad nos puede favorecer en menores requerimientos anestésicos. Mejor estado hemodinámico y por ende, menor incidencia de incrementos en la presión del LCR. Sin embargo, recordar que son pacientes con una potencial vía aérea difícil. Por lo que recomendamos si considera usar premedicación sea siempre bajo monitorización continua. Fármacos con dosis calculadas adecuadamente y siempre bajo vigilancia por personal con entrenamiento en abordaje de vía aérea difícil.

Vía aérea: Es importante considerar a estos pacientes como vía aérea difícil. Se tendrá que establecer planes alternativos de abordaje de la vía aérea de acuerdo al entrenamiento y disponibilidad de equipo o indumentaria de cada lugar. 
Nosotros recomendamos el uso de vídeo laringoscopios los cuales han demostrado mejorar el cormack-lehane además de ofrecer menos repercusión en el estado hemodinámico. Para el caso expuesto nosotros utilizamos el laringoscopio Bullard el cual quizá ya no sea considerado en los nuevos algoritmos de vía aérea difícil pero es de gran utilidad. Siempre considere que movimientos en la columna cervical podrían generar un daño adicional dependiendo del grado de lesión que estos pacientes pudiesen tener, por ende, recomendamos la estabilización de la columna cervical durante el perioperatorio sobre todo en la intubación. No existen reportes de uso de mascarillas laríngeas así que su uso deberá ser con cautela y utilizar las presiones y tiempo recomendados. Hay una evolución importante en el uso de USG para valoración de vía aérea considérela una opción de prevenir o identificar factores de riesgo.

Transoperatorio: La estabilidad hemodinámica es un punto esencial el uso de dexmedetomidina en dosis de $0,3-0,5 \mathrm{mcg} / \mathrm{kg} / \mathrm{h}$ como coadyuvante anestésico podría favorecer a dicha estabilidad además de tener acción demostrada sobre el centro de la respiración, contar con efectos analgésicos, antieméticos y de sedación. Evidentemente habrá que valorar cada caso en particular y la disponibilidad de medicamentos con los cuales contemos.

Extubación: Tome siempre en cuenta el historial de cada paciente cómo es la presencia de apneas, uso de $\mathrm{O}_{2}$ suplementario, parálisis de cuerdas vocales entre otras comorbilidades. Si el paciente no tiene ninguna contraindicación para realizar la extubación en sala de operaciones se recomienda sea en un plano profundo.

Vigilancia postoperatoria: Estos pacientes requieren una vigilancia mucho más estrecha dadas las eventualidades de la vía aérea que pueden presentarse.

Otros: La prevención del dolor y de NVPO es de vital importancia considere terapia multimodal para ambos.

\section{Conclusiones}

El conocimiento de la enfermedad, la farmacocinética y los cuidados perioperatorios constituyen la piedra angular de su manejo perioperatorio.

\section{Referencias}

1. Allepuz A. Efectividad de la sección del filum terminale para el tratamiento de la malformación de Chiari tipo I. Barcelona: Agència d'Avaluació de Tecnologia i Recerca Mèdiques. Servei Català de la Salut. Departament de Salut. Generalitat de Catalunya; 2009.

2. Adzick NS, Thom EA, Spong CY, Brock JW 3rd, Burrows PK, Johnson MP, et al. MOMS Investigators. A randomized trial of prenatal versus postnatal repair of myelomeningocele. N Engl J Med 2011 Mar;364(11):9931004.

3. AFACPA (2005-2008). "Libreto sobre Arnold-Chiari y patologías asociadas, pág. 4-10.
4. Ghaly RF, Candido KD, Sauer R, Knezevic NN. Anesthetic management during Cesarean section in a woman with residual Arnold-Chiari malformation Type I, cervical kyphosis, and syringomyelia. Surg Neurol Int 2012;3(1):26.

5. Agustí M, Adàlia R, Fernández C, Gomar C. Anaesthesia for caesarean section in a patient with syringomyelia and ArnoldChiari type I malformation. Int J Obstet Anesth 2004 Apr;13(2):114-116.

6. Carrillo-Esper R, VázquezElizondo G, Gutiérrez-Delgado LG, Guevara-Arnal L, MéndezSánchez N. Malformación de Arnold-Chiari tipo I, siringomielia, siringobulbia y atrapamiento del ventrículo
IV. Gac Med Mex 2008 JulAug;144(4):351-354.

7. Chiapparini LSV, Lazzaro C, Bruzzone M, et al. "Neuroradiological diagnosis of Chiari malformations" neurol sci, 2011; 32 (3): 283-286.

8. Dauvilliers Y, Stal V, Abril B, Coubes P, Bobin S, Touchon J et al. Chiari malformation and sleep related breathing disorders. J Neurol Neurosurg Psychiatry 2007 Dec;78(12):1344-1348.

9. Gagnadoux F, Meslier N, Svab I, Menei P, Racineux JL. Sleepdisordered breathing in patients with Chiari malformation: improvement after surgery. Neurology 2006 Jan;66(1):136-138.

10. Castro-Cordero HAJ, Avendaño J. "Manejo quirúrgico de los casos de siringomielia de unión 
craneocervical, cervical y dorsal en el Instituto Nacional de Neurología y Neurocirugía" neurociencias de México, 2011; 16(2): 47-49.

11. Eule JM, Erickson MA, O’Brien MF, Handler M. Chiari I malformation associated with syringomyelia and scoliosis: a twenty-year review of surgical and nonsurgical treatment in a pediatric population. Spine 2002 Jul;27(13):1451-1455.

12. Martínez-Sabater A, SanchoCantus D. Malformación de Arnold-Chiari y siringomielia en atención primaria. A propósito de un caso. Semergen 2011; http://dx.doi.org/10.1016/j.se- merg.2011.09.007.

13. Wan MJ, Nomura $\mathrm{H}$, Tator $\mathrm{CH}$. Conversion to symptomatic Chiari I malformation after minor head or neck trauma. Neurosurgery 2008 Oct;63(4):748753.

14. Bejjani GK. Definition of the adult Chiari malformation: a brief historical overview. Neurosurg Focus 2001;11(1):E1.

15. Imperato A, Seneca V, Cioffi V, Colella G, Gangemi M. Treatment of Chiari malformation: who, when and how. Neurol Sci 2011 Dec;32(S3 Suppl 3):S335-S339.

16. Mottolese C, Szathmari A, Simon E. Treatment of Chiari type
I malformation in children: the experience of Lyon Neurol Sci, 2011; 32:325-330, DOI http:// dx.doi.org/10.1007/s10072-0110685-2.

17. Eldredge EA. Soriana, Rockoff MA. Pediatric neurosurgical anesthesia. In: Cote CJ, Todres ID, Goudsouzian NG, Ryan JF (Eds). A Practice of Anesthesia for Infants \& Children; 3rd ed. WB Saunders 2001; 493-521.

18. Setz AC, De Boer HD, Driessen JJ, Scheffer GJ. Anesthetic management in a child with ArnoldChiari malformation and bilateral vocal cord paralysis. Paediatr Anaesth 2005 Dec;15(12):11051107. 